\title{
Surface Wave Energy from Point Sources in Plane Layered Earth Models ${ }^{1}$
}

\author{
David G. Harkrider ${ }^{2}$ and Don L. Anderson \\ Seismological Laboratory \\ California Institute of Technology, Pasadena
}

\begin{abstract}
The total energy contained in a surface wave can be computed from its propagation-corrected spectrum by integrating over the surface of the earth and over the depth of the wave guide. The former requires knowledge of the radiation pattern of the source. The latter requires only a knowledge of the variation of physical properties with depth. In this paper the depth integration is performed for a continental and an oceanic earth model for three Rayleigh modes and four Love modes. The results are presented in tables and graphs in such a way that it is convenient to convert an observed surface wave displacement or displacement spectrum to total energy density. If the surface radiation pattern is known, the surface integration then yields the total energy in the observed spectrum. The partioning of energy between surface wave modes is computed for several simple sources at the surface and at depth, making it possible to estimate the energy contained in frequency bands or modes which are inaccessible for direct analysis. The increasing importance of the higher modes in the total energy budget at short periods and for channel depth sources is demonstrated. The shapes of the spectrums are diagnostic of source orientation and depth.
\end{abstract}

\section{INTRODUCTION}

Energy is an important concept in both theoretical and experimental seismology. It is involved in the derivation of the wave equation and in the variational methods of theoretical analysis. It has recently found application in the universal dispersion theory [Anderson, 1964], the earth anelasticity theory [Anderson and Archambeau, 1964], the inversion theory [Archambeau and Anderson, 1963], and the analytic calculation of group velocity [Jeffreys, $1961]$.

Energy radiation and energy partition of surface waves are important to our understanding of properties of the seismic source such as magnitude, mechanism, and depth. Even more fundamental is the question of energy balance of the earth. It has never been possible to measure all of the energy radiated in all directions at all frequencies from a single earthquake, much less the total annual tectonic energy release. It will never be possible, of course, to account for all the energy radiated by an earthquake, but the

\footnotetext{
${ }^{1}$ Contribution 1386, Division of Geological Sciences, California Institute of Technology, Pasadena.

${ }^{2}$ Now at Brown University, Providence, Rhode Island.
}

present methods of estimating energy from the magnitude are clearly unsatisfactory.

In this paper we compute the partition of energy among various surface wave modes for horizontal and vertical point sources at various depths in realistic earth models. Basic to this task is the excitation theory of Harkrider [1964].

In addition to explaining the presence or $a b-$ sence of individual modes and their relative energies, the theoretical results allow us to estimate the energy in frequency bands and modes that are not accessible for direct measurement. The partition functions and observed surface amplitudes can then be used in calculating the minimum seismic energy associated with an earthquake. Magnitudes and magnitude scales will have to be consistent with this observed minimum energy. Also, the strain energy release of a proposed source mechanism must be able to account for the observed seismic energy release.

In this paper we give expressions for the surface wave spectral energy density for various source depths and orientations and obtain the short- and long-period asymptotes. Relations are also given for obtaining the necessary medium response functions in terms of normalized energy integrals. These expressions are then 
evaluated and tabulated for a continental and an oceanic earth model.

The energy integrals given in this paper are useful in calculating a great variety of important seismic parameters, such as:

(a) Accurate values of the group velocity and the variation of relative excitation with frequency and mode.

(b) The effect of any parameter in any layer on the phase velocity [Anderson, 1964].

(c) The attenuation of surface waves, given the variation of anelasticity with depth [Anderson and Archambean, 1964; Anderson et al., $1965]$.

(d) The inverse of the two preceding problems.

(e) The energy for individual modes of surface waves from the observed or known spectrum of an identifiable mode.

(f) The energy partition as a function of depth of surface wave modes for seismic sources at the surface and at depth.

\section{Symbols.}

$T^{R}, T^{L}$, Rayleigh and Love kinetic energy density integrals, normalized to surface displacements.

$V^{R}, V^{L}$, Rayleigh and Love potential energy density integrals, normalized to surface displacements.

$\omega$, angular frequency.

$k_{R}, k_{L}$, Rayleigh and Love angular wave numbers. $z$, vertical or depth coordinate.

$I_{0}{ }^{R}, I_{1}{ }^{R}, I_{2}{ }^{R}, I_{3}{ }^{R}$, depth integrations defined by (3).

$I_{0}{ }^{L}, I_{1}{ }^{L}, I_{2}{ }^{L}$, depth integrations defined by (4).

$Q, W$, normalized horizontal and vertical Rayleigh displacements.

$V$, normalized Love displacements.

$\dot{u}^{*}(z) / \dot{w}_{0}, \dot{v}(z) / \dot{v}_{0}, \dot{w}(z) / \dot{w}_{0}$, Haskell's plane wave particle velocity (or displacement) ratios at depth $z$.

$\sigma_{R}{ }^{*}(z)$, Haskell's plane wave normal stress factor at depth $z$.

$\tau_{R}(z), \tau_{L}^{*}(z)$, Haskell's Rayleigh and Love tangential stress factors at depth $z$.

$d_{j}$, thickness of layer $j$.

$\alpha_{j}$, compressional velocity of layer $j$.

$\beta_{i}$, shear velocity of layer $j$.

$\rho_{j}$, density of layer $j$.

$\mu_{i}$, rigidity of layer $j$.

$\lambda_{i}$, Lame's constant of layer $j$.
$C_{R}, C_{L}$, Rayleigh and Love phase velocities.

$U_{R}, U_{L}$, Rayleigh and Love group velocities.

$A_{R}, A_{L ;}$ Rayleigh and Love medium response. functions.

$\mu_{P}, \rho_{P}, \beta_{P}$, composite plate rigidity, density, and velocity defined by (14).

$V_{R j}$, Rayleigh velocity of layer $j$.

$\gamma_{i}$, layer $j$ coefficient defined by (16).

$E^{R}, E^{L}$, Rayleigh and Love spectrum energy densities per unit propagation path.

$r$, epicenter distance.

$\theta$, azimuth angle.

$h$, source depth.

$\left[w_{0},(r, \theta, h)\right]_{R}$, vertical spectral surface displacement for Rayleigh waves.

$\left[v_{0}(r, \theta, h]_{L}\right.$, horizontal spectral surface displacement for Love waves.

$\langle L\rangle$, spectral source strength.

$E_{V}{ }^{R}, E_{V}{ }^{L}$, Rayleigh and Love spectrum energy densities per unit propagation path for a vertical force.

$E_{H} R, E_{H}{ }^{L}$, Rayleigh and Love spectrum energy densities per unit propagation path for horizontal force.

\section{Fundamentat Formulas}

The basic integrals for kinetic and potential energy density, normalized to the surface displacement, are given by

$$
\begin{gathered}
2 T^{R}=\omega^{2} I_{0}{ }^{R} \\
2 V^{R}=k_{R}{ }^{2} I_{1}{ }^{R}+2 k_{R} I_{2}{ }^{R}+I_{3}{ }^{R}
\end{gathered}
$$

for Rayleigh waves and

$$
\begin{gathered}
2 T^{L}=\omega^{2} I_{0}{ }^{L} \\
2 V^{L}=k_{L}{ }^{2} I_{1}{ }^{L}+I_{2}{ }^{L}
\end{gathered}
$$

for Love waves, with

$$
\begin{aligned}
& I_{0}{ }^{R}=\int_{0}^{\infty} \rho\left(Q^{2}+W^{2}\right) d z \\
& I_{1}{ }^{R}=\int_{0}^{\infty}\left\{(\lambda+2 \mu) Q^{2}+\mu W^{2}\right\} d z \\
& I_{2}{ }^{R}=\int_{0}^{\infty}\left\{\lambda Q \frac{d W}{d z}-\mu W \frac{d Q}{d z}\right\} d z \\
& I_{3}{ }^{R}=\int_{0}^{\infty}\left\{(\lambda+2 \mu)\left(\frac{d W}{d z}\right)^{2}+\mu\left(\frac{d Q}{d z}\right)^{2}\right\} d z
\end{aligned}
$$

and 


$$
\begin{aligned}
& I_{0}{ }^{L}=\int_{0}^{\infty} \rho V^{2} d z \\
& I_{1}{ }^{L}=\int_{0}^{\infty} \mu V^{2} d z \\
& I_{2}{ }^{L}=\int_{0}^{\infty} \mu\left(\frac{d V}{d z}\right)^{2} d z
\end{aligned}
$$

deffreys, 1961]. The normalized displacements and displacement derivatives in the integrands of (3) and (4) can be expressed in terms of the Thomson-Haskell layer vector elements [Haskell, 1953]:

$$
\begin{gathered}
Q=\dot{u}^{*}(z) / \dot{w}_{0} \\
W=\dot{w}(z) / \dot{w}_{0} \\
\frac{d Q}{d z}=\frac{k_{R}}{\mu}\left\{\mu\left[\frac{\dot{w}(z)}{\dot{w}_{0}}\right]+\left[\frac{\tau_{R}(z) C_{R}}{\dot{w}_{0}}\right]\right\} \\
\frac{d W}{d z}=-\frac{k_{R}}{(\lambda+2 \mu)} \\
\cdot\left\{\lambda\left[\frac{\dot{u}^{*}(z)}{\dot{w}_{0}}\right]+\left[\frac{\sigma_{R}^{*}(z) C_{R}}{\dot{w}_{0}}\right]\right\}
\end{gathered}
$$

and

$$
\begin{aligned}
V & =\dot{v}(z) / \dot{v}_{0} \\
d V / d z & =-\left(k_{L} / \mu\right)\left[\tau_{L}{ }^{*}(z) C_{L} / \dot{v}_{0}\right]
\end{aligned}
$$

for a layered half-space composed of $n$ elastic layers, where $\lambda_{j}=\rho_{j}\left(\alpha_{j}{ }^{2}-2 \beta_{j}{ }^{2}\right)$ and ${ }_{j} \xi_{j}=\rho_{j} \beta_{j}{ }^{2}$. For computing purposes the relations between the vector elements, the phase velocities $C_{z}$ and $C_{z}$, and the Thomson-Haskell layer matrices can be found in Harkrider [1964].

The Thomson-Haskell matrix technique has been used in two ways to evaluate the energy integrals. $W u$ [1966] used the matrix method in calculating values of the integrands at various depths for the fundamental modes and then computed the kinetic energy in integrals of (1) and (2) by numerical integration. Anderson [1964] used the matrix-calculated eigenfunctions in an analytic evaluation of the Love wave integrals. Anderson's technique is used in this paper.

The energy integrals can be used in calculating group velocity and the medium response without differentiation. Group velocity is determined by equating the kinetic and potential energy expressions in (1) and (2) and then invoking Rayleigh's principle [Meissner, 1926; Jeffreys, 1934, 1961]. The resulting expressions for group velocity are

$$
\begin{gathered}
U_{R}=\left(I_{1}^{R}+I_{2}^{R} / k_{R}\right) /\left(C_{R} I_{0}{ }^{R}\right) \\
U_{L}=I_{1}{ }^{L} /\left(C_{L} I_{0}{ }^{L}\right)
\end{gathered}
$$

This technique has been extended to group velocity calculations for spherical earth models by Takeuchi et al. $[1962,1964]$ and Kovach and Anderson [1964].

A modification of this technique allows one to calculate the changes in Rayleigh and Love wave phase velocities resulting from changes in the physical properties of the wave guide [Jeffreys, 1961; Anderson, 1964; Takeuchi et al., 1964]. These perturbations can, in turn, be used for the calculation of the attenuation of surface waves as a function of period for realistic anelastic earth models [Anderson and Archambeau, 1964; Anderson et al., 1965].

Integral relations similar to (7) and (8) can also be obtained for the medium response. Comparing the two-dimensional or lime-source solutions of Neigaus [Kellis-Borok and Yanovskaya, 1962] with the three-dimensional or pointsource solutions of Harkrider [1964], we see that the medium response can be expressed as

$$
\begin{gathered}
A_{R}=\left[2 C_{R} U_{R} I_{0}{ }^{R}\right]^{-1} \\
=\left[2\left(I_{1}{ }^{R}+I_{2}{ }^{R} / k_{R}\right)\right]^{-1} \\
A_{L}=\left[2 C_{L} U_{L} I_{0}{ }^{L}\right]^{-1}=\left[2 I_{1}{ }^{L}\right]^{-1}
\end{gathered}
$$

It is interesting that in $(7),(8),(9)$, and (10) we are able to express the group velocity and the medium response in terms of the normalized energies which are used to calculate phase velocity perturbations [Anderson, 1964; Takeuchi et al., 1964]. These relations make it convenient to calculate partial derivatives, not only for phase velocity but for the complete spectral response of any reasonable structure due to a change of elastic parameter at depth.

By using analytical expressions for the depth integrations, we were able to derive the shortand long-period limits for the fundamental mode Rayleigh and Love wave energy integrals. For high frequencies, the asymptotic form of $I_{0}{ }^{L}$ is

$$
I_{0}{ }^{L} \rightarrow d_{1} \rho_{1} / 2
$$


TABLE 1. Layer parameters for the oceanic and shield earth models.

Ocean

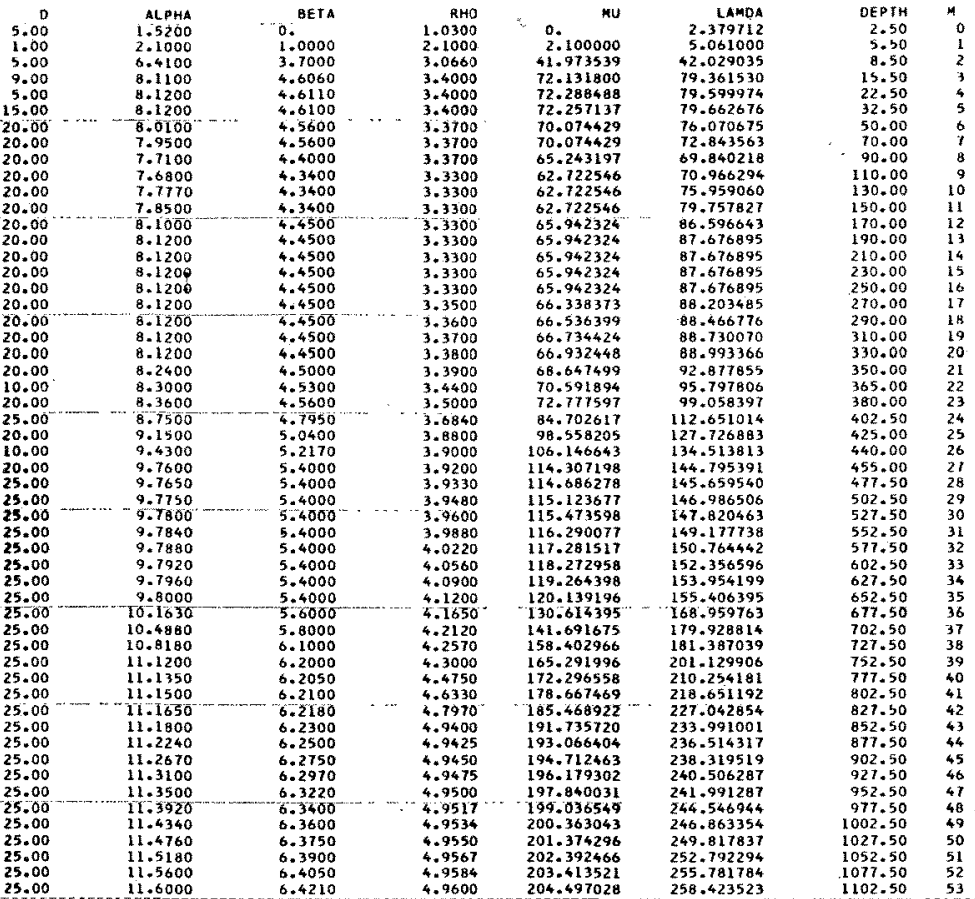

Shield

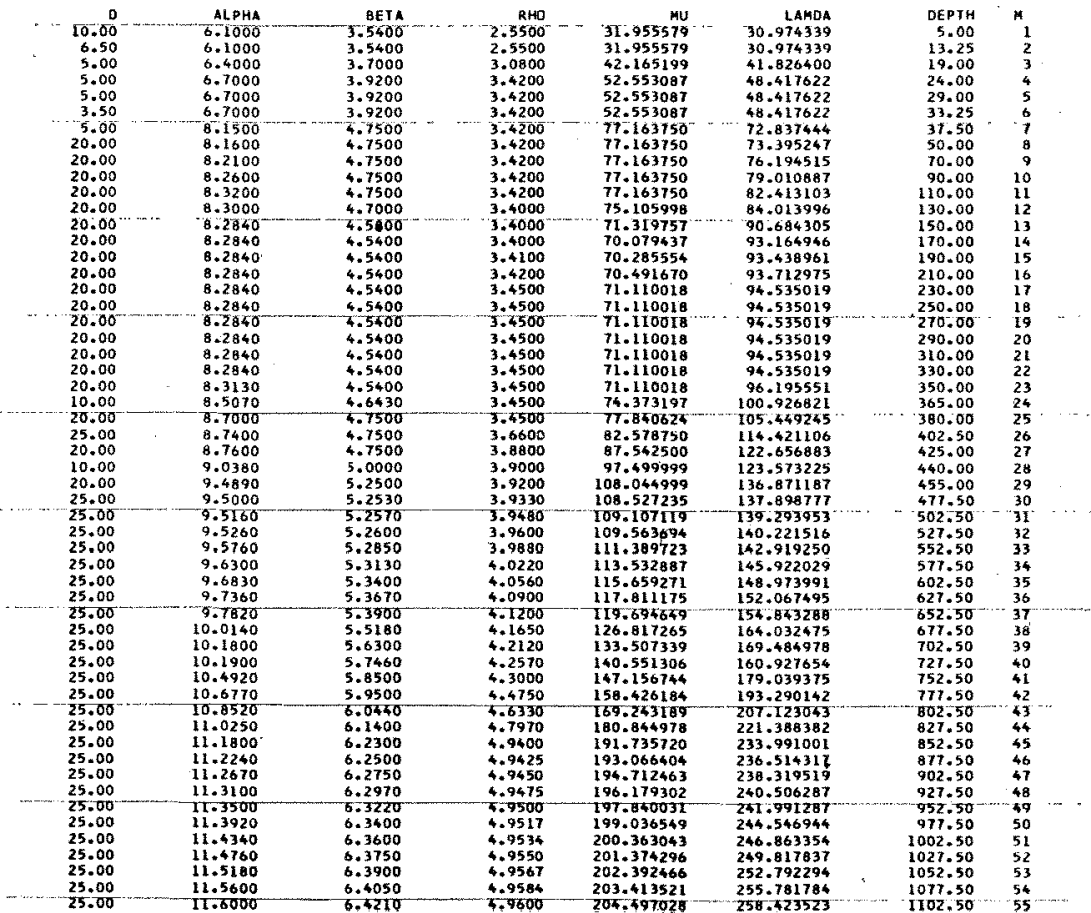




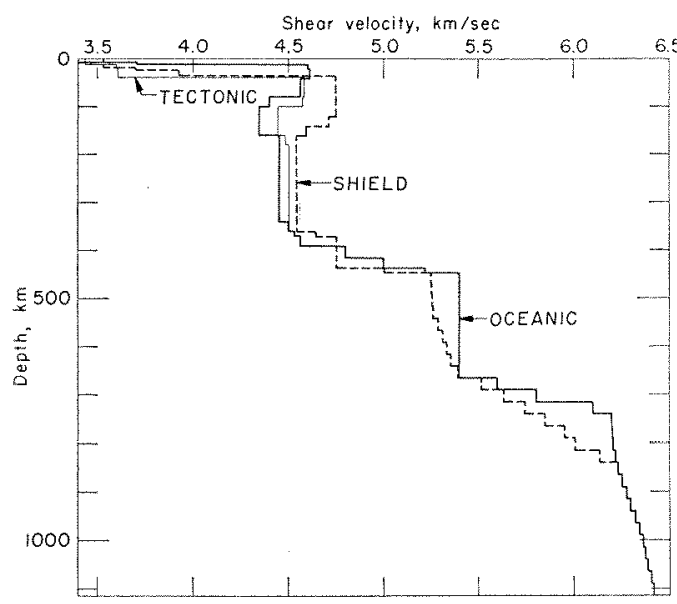

Fig. 1. Shear velocity as a function of depth for oceanic, tectonic, and shield earth models [Anderson and Toksöz, 1966; Anderson 1966].

and from (10)

$$
A_{L}=\left(\mu_{1} d_{1}\right)^{-1}
$$

since $C_{L} \rightarrow U_{L} \rightarrow \beta_{1}$ as $\omega \rightarrow \infty$. For long periods of the fundamental Love mode, $I_{0}{ }^{2}$ can be expressed in the limit as

$$
I_{0}{ }^{L} \rightarrow \mu_{n}{ }^{2} /\left[2 \omega^{2} d_{p} \mu_{p}\left(\beta_{n}^{2} / \beta_{p}^{2}-1\right)\right]
$$

where the subscript 1 designates the surface layer and $n$ the $n$th layer or half-space. The new quantities are defined by

$$
\begin{aligned}
& \mu_{p}=\sum_{j=1}^{n-1} \frac{\mu_{j} d_{j}}{d_{p}} \\
& \rho_{p}=\sum_{j=1}^{n-1} \frac{\rho_{j} d_{j}}{d_{p}} \\
& d_{p}=\sum_{j=1}^{n-1} d_{j}
\end{aligned}
$$

and $\beta_{p}{ }^{2}=\mu_{p} / \rho_{p}$

From (10) we obtain the long-period limit

$$
A_{L}=\omega^{2} d_{p} \mu_{p}\left(\beta_{n}{ }^{2} / \beta_{p}{ }^{2}-1\right) /\left(\beta_{n} \mu_{n}\right)^{2}
$$

using the limits

$$
C_{L} \rightarrow U_{L} \rightarrow \beta_{n} \quad \text { as } \quad \omega \rightarrow 0
$$

For Rayleigh waves the high-frequency and long-period asymptotes are

$I_{0}{ }^{R} \rightarrow 2 V_{R i} \rho_{j} D_{j} / \omega\left(1-V_{R i}{ }^{2} / \alpha_{j}{ }^{2}\right)^{1 / 2}$ where

$$
\begin{gathered}
D_{j}=\beta_{j}{ }^{2} \gamma_{j}{ }^{2}\left(\alpha_{j}{ }^{2}+\beta_{i}{ }^{2}\right. \\
\left.-2 V_{R i}{ }^{2}\right) /\left[\alpha_{i} V_{R j}\left(\gamma_{j}-1\right)\right]^{2}-\left(\gamma_{i}-1\right) \\
\gamma_{j}=2\left(\beta_{i} / V_{R i}\right)^{2}
\end{gathered}
$$

and from (10)

$$
A_{R} \rightarrow \omega\left(1-V_{R j}{ }^{2} / \alpha_{j}{ }^{2}\right)^{1 / 2} /\left(4 \rho_{i} V_{R i}{ }^{3} D_{j}\right)
$$

since $C_{n} \rightarrow U_{R} \rightarrow V_{R}$, with the subscript $j=1$ for $\omega \rightarrow \infty$ and $j=n$ for $\omega \rightarrow 0$. This shortperiod expression is only valid for an all solid model; for oceanic models the limit is different and is not given here.

Expressions 12, 15, and 17 are identical with the limits given by Harkrider [1966], which were obtained by taking the short- and longperiod limits of the residue evaluation of the solutions for buried sources.

Since the kinetic and potential energy integrals of (1) and (2) are equal for surface waves, we can write the spectral energy densities per unit propagation path as

$E^{R}=\int_{0}^{2 \pi} \omega^{2} I_{0}{ }^{R}\left|\left[w_{0}(r, \theta, h)\right]_{R}\right|^{2} r d \theta$

$E^{L}=\int_{0}^{2 \pi} \omega^{2} I_{0}{ }^{L}\left|\left\{V_{0}(r, \theta, h)\right\}_{L}\right|^{2} r d \theta$

Evaluating these expressions with the spectral solutions for buried sources in a multilayered medium [Harkrider, 1964; Ben-Menahem and Harkrider, 1964], we obtain at distances large in comparison with the wavelength

$$
\begin{aligned}
{E_{V}}^{R} & =\omega\langle L\rangle^{2} C_{R} A_{R}{ }^{2} I_{0}{ }^{R}\left[\dot{w}(h) / \dot{w}_{0}\right]^{2} \\
E_{V}{ }^{L} & \equiv 0
\end{aligned}
$$

for a vertical source at depth $h$ and

$$
\begin{gathered}
{E_{H}{ }^{R}}^{R}=(\omega / 2)\langle L\rangle^{2} C_{R} A_{R}{ }^{2} I_{0}{ }^{R}\left[\dot{u}^{*}(h) / \dot{w}_{0}\right]^{2} \\
E_{H}{ }^{L}=(\omega / 2)\langle L\rangle^{2} C_{L} A_{L}{ }^{2} I_{0}{ }^{L}\left[\dot{v}(h) / \dot{v}_{0}\right]^{2}
\end{gathered}
$$

for a horizontal source at the same depth with source strength $\langle L\rangle$.

\section{Numerical Results}

The numerical calculations presented in this paper are for two extreme models of the earth's mantle structure. The oceanic and shield models are given in Table 1 and Figure 1. These structures were derived by applying the universal 
TABLE 2. Normalized Rayleigh and Love energy integrals in units of $10^{\circ}$ ergs/ $\mathrm{cm}^{2}$ for the ocean structure.

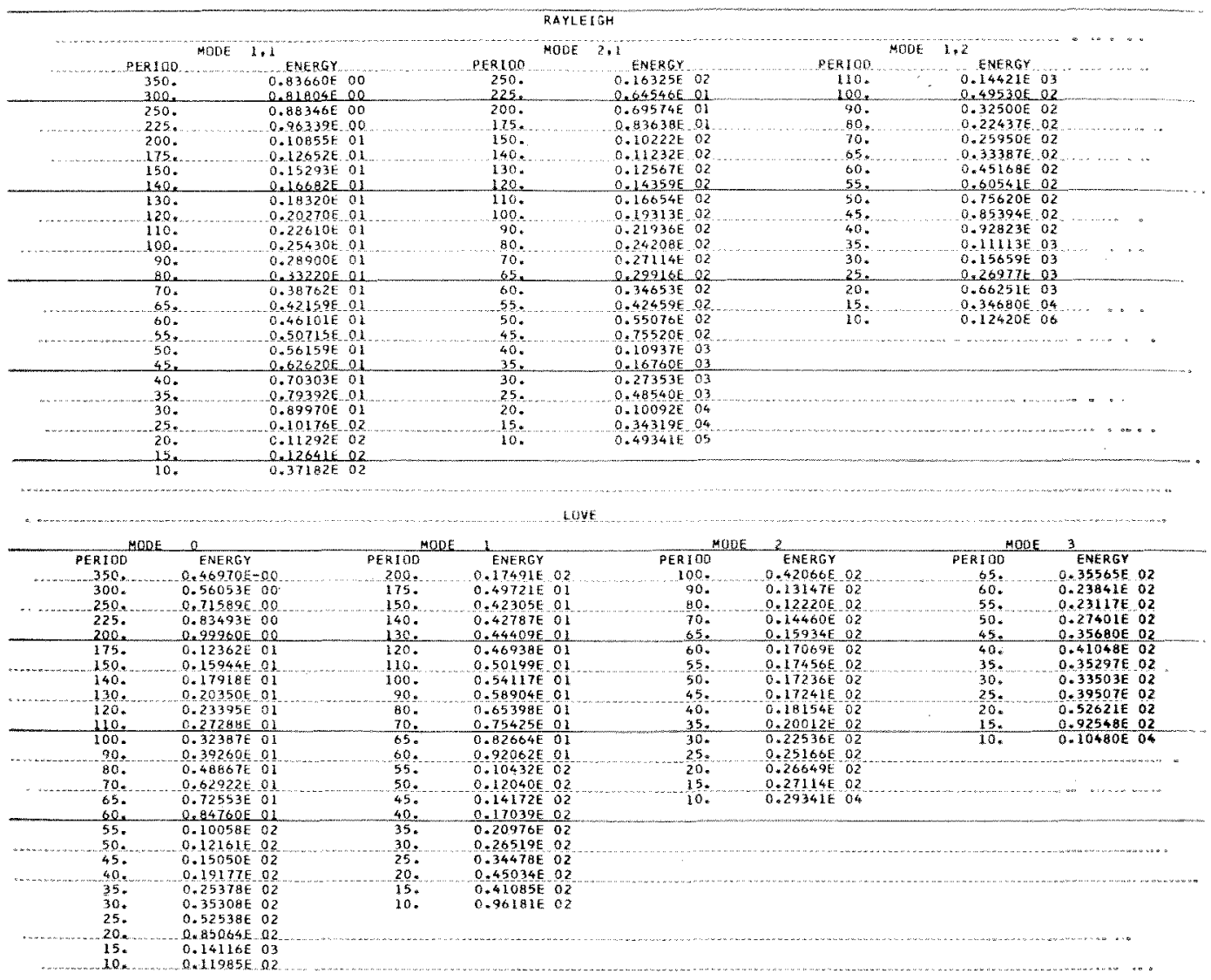

dispersion theory of Anderson [1964] to the great-circle data of Tolusöz and Anderson [1966]. Approximately 53 layers are used in each case to define the mantle structure.

For each model we provide in tables and/or figures the basic quantities necessary for calculating the surface wave energies for the first three Rayleigh and the first fou Love modes.
The effect of source depth is presented for the oceanic model, but tables for including the source depth can be found in Harkrider [1966] for a much wider range of depths for both models.

It is advisable at this point to mention some of the basic differences between the two structural models. The most pronounced diference 


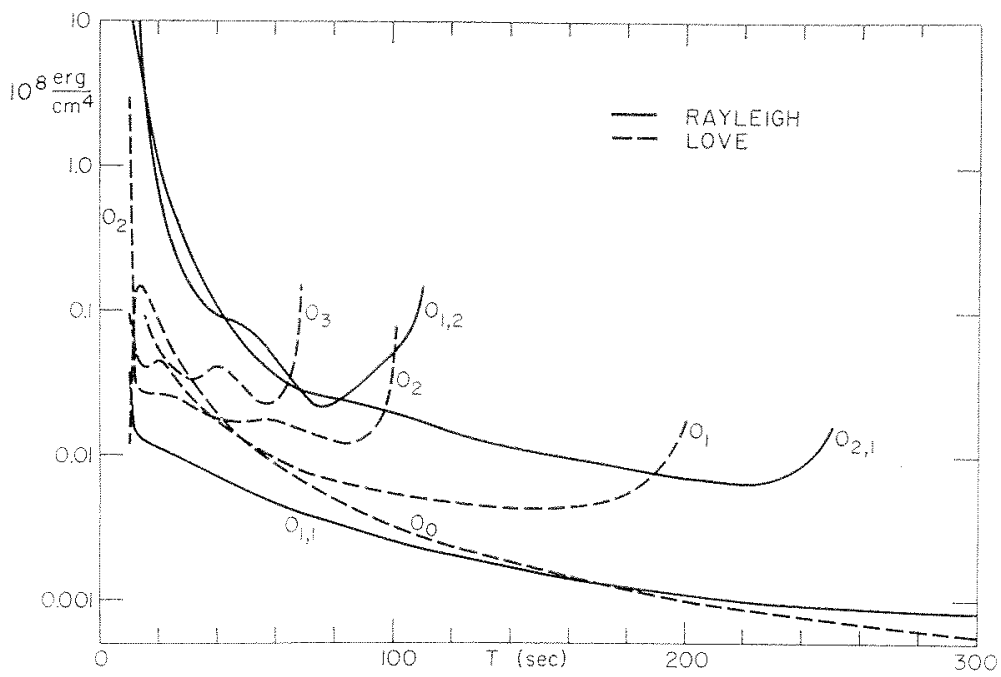

Fig. 2. Nomalized Love and Rayleigh energy density integrals for the oceanic structure.

(Figure 1) occurs in the mantle low-velocity zone between 100 and $400 \mathrm{~km}$. The oceanic nodel has a shallower and more pronounced low-veloeity zone in addition to a thimner custal wave guide. Thus, if any differences between models occur in the numerical results, we would expect them to be most apparent in the trapped modes, i.e. Love waves and the highermode Rayleigh waves. These are waves whose very existence depends on the presence of wave guides either at the surface or at depth, and they are thus more sensitive to the details of wave guides than the fundamental Rayleigh wave. The two pronounced upper mantle discontinuities at depths near 400 and $700 \mathrm{~km}$ are important features of recent conclusions regarding surface wave dispersion.

For the oceanic model, the sum of the normalized kinetic and potential energy density integrals are given in Table 2 and Figure 2. Since these two integrals are equal, the curves represent $2 T^{20}$ and $2 T^{2}$, given in (1) and (2). The same quantities are given for the shield model in Table 3 and Figure 3. From the limits (16) we see that the energy density integral for fundamental Rayleigh waves is inversely prom portional to period at both the long and short periods. For the fundamental Love wave, the limits (11 and 13) require that the energy density integral approach a constant value at long periods and be inversely proportional to the square of the period at short periods.
From the figures we see that the long-period asymptotic behavior of the energy integrals is beyond the range of the tabulated results. In fact, on the basis of the figures alone, we might suspect that the quoted long-period trends of Love and Rayleigh waves had been interchanged. To verify the limits, we calculated the energy for very long periods, and the results agree with the theory.

At short periods the asymptotic trends of the Rayleigh and Love fundamental modes for the shield model can be seen in Figure 3. The relatively complicated behavior (Figure 2) at short periods is associated with the surface, crustal, and upper mantle wave guides. Complications occur at shorter periods for the oceanic model because of the thinner crust and shallower lowvelocity zone.

As suggested earlier, the contrast between models is greatest in the trapped modes, especially the Love modes. Very little difference in the normalized energy densities of the fundamental Rayleigh waves occurs over the range of calculated periods.

Energy curves obtained by numerical integration can be found for additional earth models in $W u$ [1966].

To calculate the actual variation of energy with frequency and mode, we must operate on the normalized energies with the spectrum of surface displacements obtained from a given source. 
TABLE 3. Normalized Rayleigh and Love energy integrals in units of $10^{\mathrm{m}} \mathrm{ergs} / \mathrm{cm}^{4}$ for the shield structure.

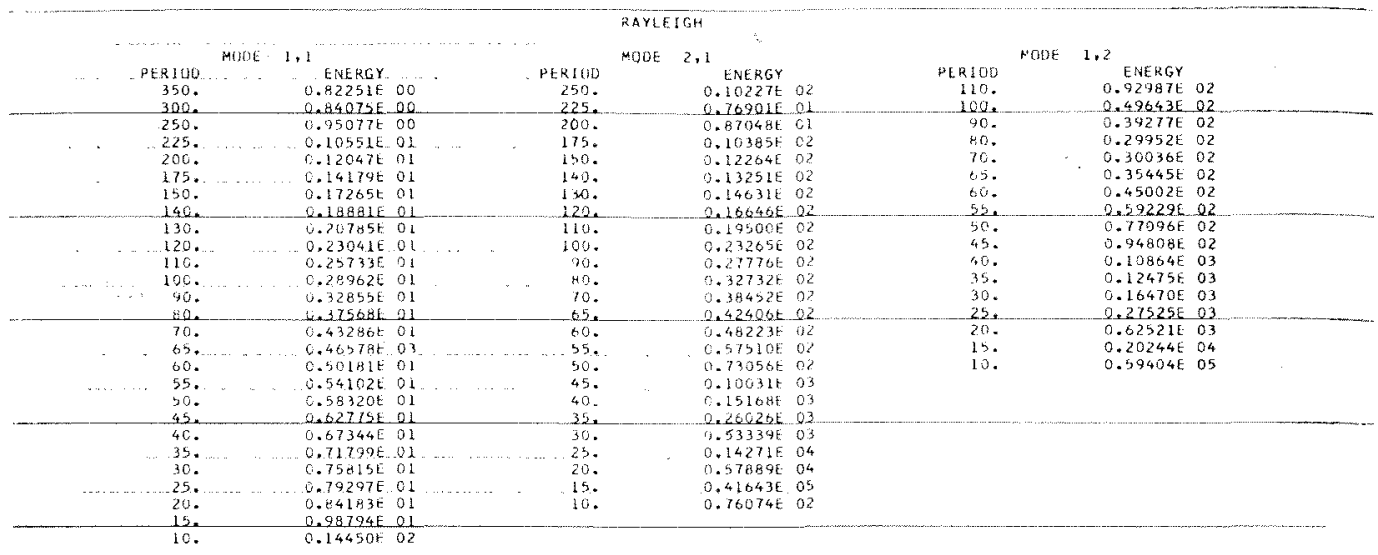

Euve

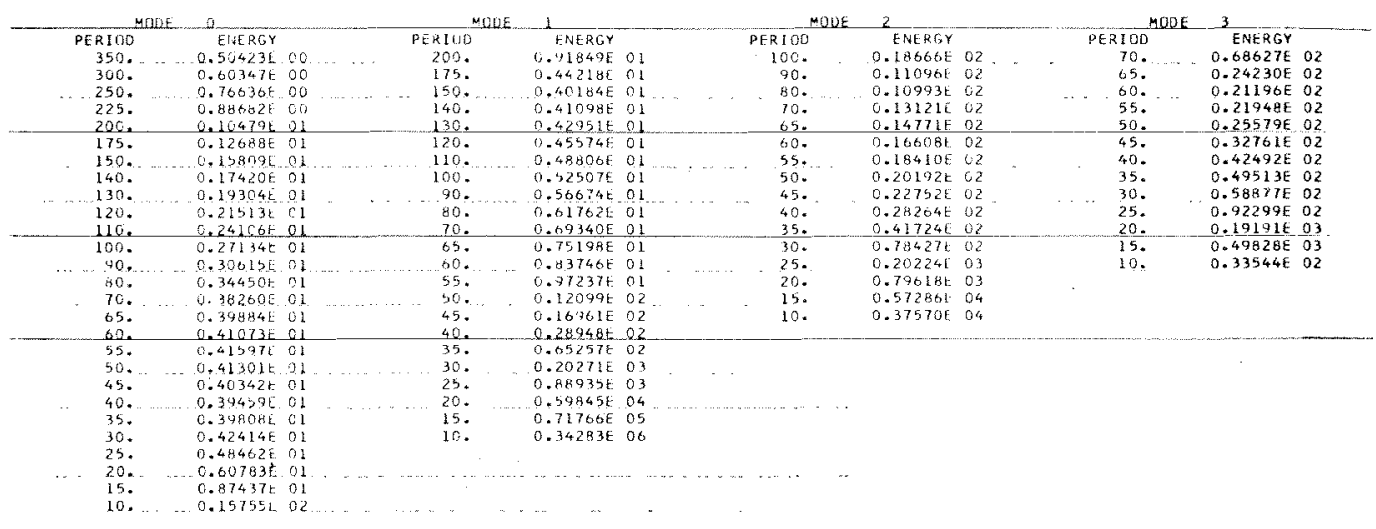

To facilitate computation and comparison, we adopt as a standard spectrum that due to a surface point source. The spectral density of surface displacement due to a surface source is referred to as relative excitation in this paper. The relative excitations for Rayleigh and Love waves for the oceanic and shield models are given in Figures 4 and 5 .

As with the normalized energy integrals, there is little difference between the two models in the relative excitation of fundamental Rayleigh waves from 300 to 20 seconds. The major differences occur in the trapped modes, especially the Love waves. The shift in amplitude toward the high frequencies in the oceanic relative to the shield model is presumably due to the thinner oceanic wave guide with the maximums for Love waves shifted approximately from 50 


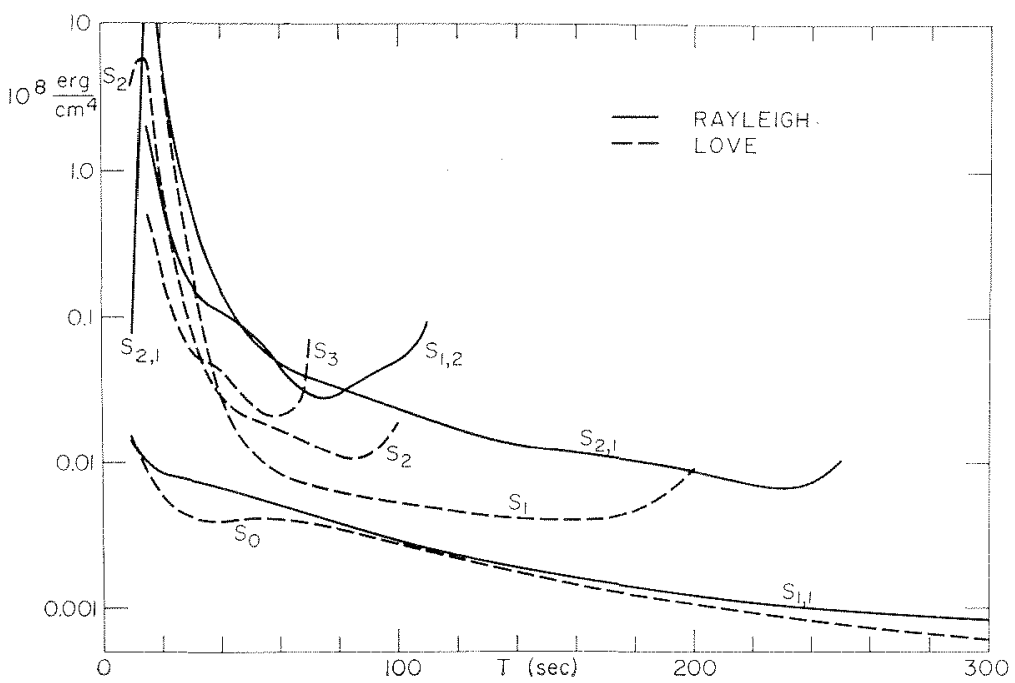

Fig. 3. Normalized Love and Rayleigh energy density integrals for the shield structure.

to 20 seconds. Note the increasing importance of the higher modes relative to the fundamental mode at intermediate periods.

Physically, these curves are the response of the medium to a surface source. Thus we would expect the amplitude of the trapped modes to become much smaller than the fundamental Rayleigh mode at low and high frequencies. This is reasonable because, at these limits, we are approaching half-space conditions for which Love waves and higher modes do not exist.

In Figure 6 we show the Rayleigh and Love wave energy densities per unit propagation path for surface sources at different orientations for oceanic and shield structures. In the figures, the ingle-subscripted dashed curves are Love modes, and the double-subscripted solid curves refer to Rayleigh modes. A vertical source, of course, does not generate Love waves.

In all cases the energy of the higher-mode Rayleigh waves is at least an order of magnitude less than that contained in the fundamental Rayleigh mode. This is in direct contrast to the normalized energies (Figures 2 and 3) where the opposite was true. Also, the energy in higher modes drops at both the long and short periods and, in all modes, at the long periods. The energies contained in the fundamental Love and Rayleigh modes are roughly comparable for a horizontal surface source, and the higher Love modes are much more energetic than the corresponding Rayleigh modes.
The importance of the short-period surface waves in any estimate of total surface wave energy is obvious from these figures. From (20), (22), and (23) we see that at long periods the fundamental Rayleigh mode is of the order of $\omega^{2}$ and the fundamental Love mode is of the order $\omega^{3}$. The greater energy in the trapped modes of the oceanic model relative to the shield model for a surface source reflects the relative nearness to the surface of the oceanic wave guides. As noted earlier for other spectral quantities, the energies for the fundamental Rayleigh mode are remarkably similar for the two models.

For a horizontal surface force, the energy density of the fundamental Rayleigh mode decreases by a factor of about 4 . This can be seen quantitatively by comparing (20) with (22), since the square of surface ellipticity for fundamental Rayleigh waves is about 0.5 for a wide range of periods.

An assumption of equipartition of energy density between all surface wave modes is obviously not valid even for estimation purposes. On the other hand, there is to some degree an equipartition of energy density between the fundamental Rayleigh and fundamental Love modes for a horizontal surface source. This is true for both models. Also, as noted above, the higher Love modes contain more energy than the higher Rayleigh modes.

For certain frequencies the energy densities 


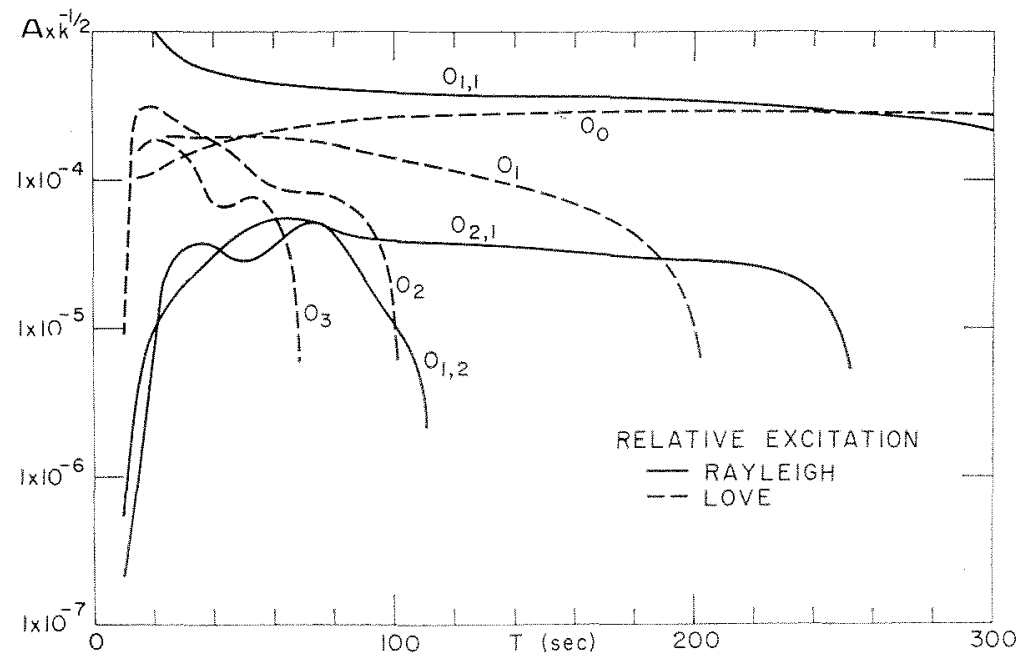

Fig. 4. Relative spectral exeitation for the oceanic structure in units of $10^{-12.8} \mathrm{~cm}^{3 / 2} /$ dyne.

of higher-mode Rayleigh waves are approximately equal, and similarly for the higher-mode Love waves. Thus, in these frequency bands, a rough equipartition of energy density is obtained for the higher modes of Rayleigh and Love waves. For the oceanic model the equipartition includes the fundamental Love wave with its higher modes.

The effect of source depth on the first three Rayleigh modes for a vertical force in an oceanic model is illustrated in Figure 7 . The figure at the top left is for the surface source given in Figure 6 and is used as a reference. At the top right we show the spectral energy density per unit propagation path for the fundamental mode for a surface focus and for three other source depths.

In general, increasing the depth decreases the amount of energy in the fundamental mode. However, a focus at $50 \mathrm{~km}$, which is the top of the low-velocity zone, can generate Rayleigh waves with slightly more energy for certain frequencies than a surface source. The higher modes all have critical depths at which they are excited most efficiently and also periods at which they are not excited at all. For a given

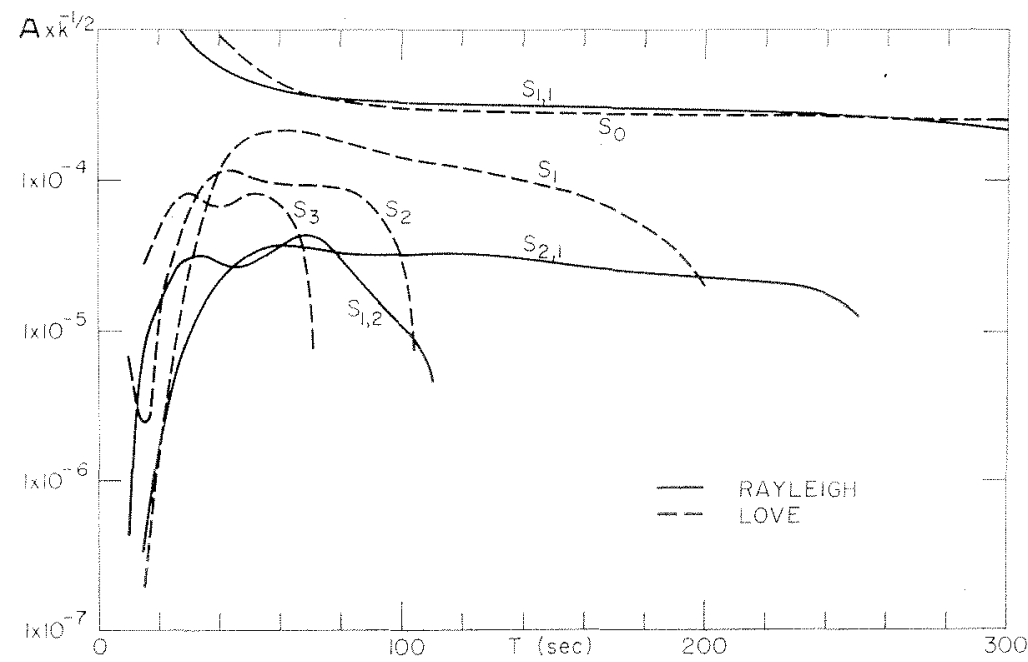

Fig. 5. Relative spectral excitation for the shield structure in units of $10^{-15.5} \mathrm{~cm}^{3 / 2} /$ dyne. 

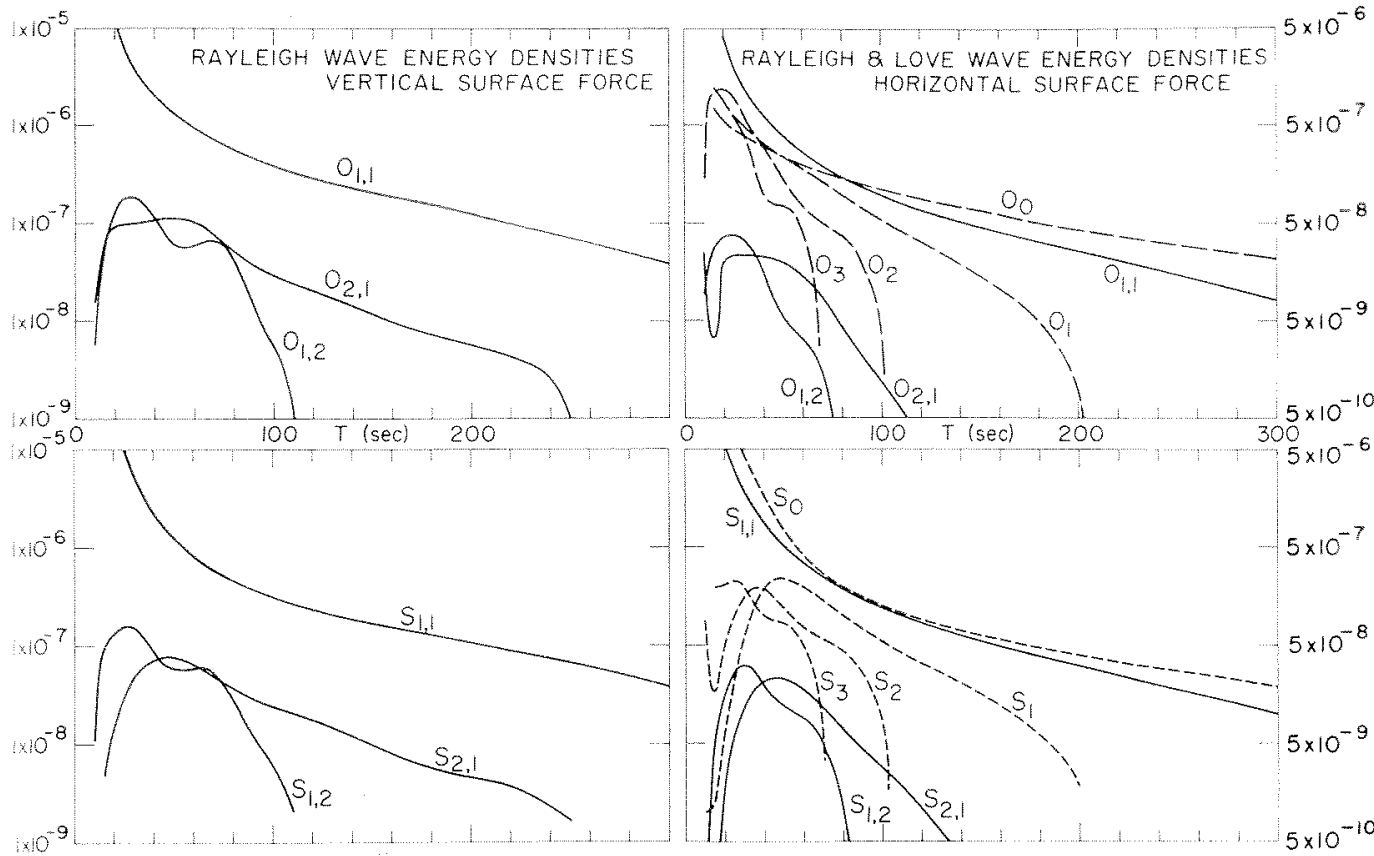

Fig. 6. Spectral energy densities per unit propagation path in units of $10^{15} \mathrm{ergs} / \mathrm{km}$ for a surface force of strength $\langle L\rangle=10^{43}$ dynes sec.

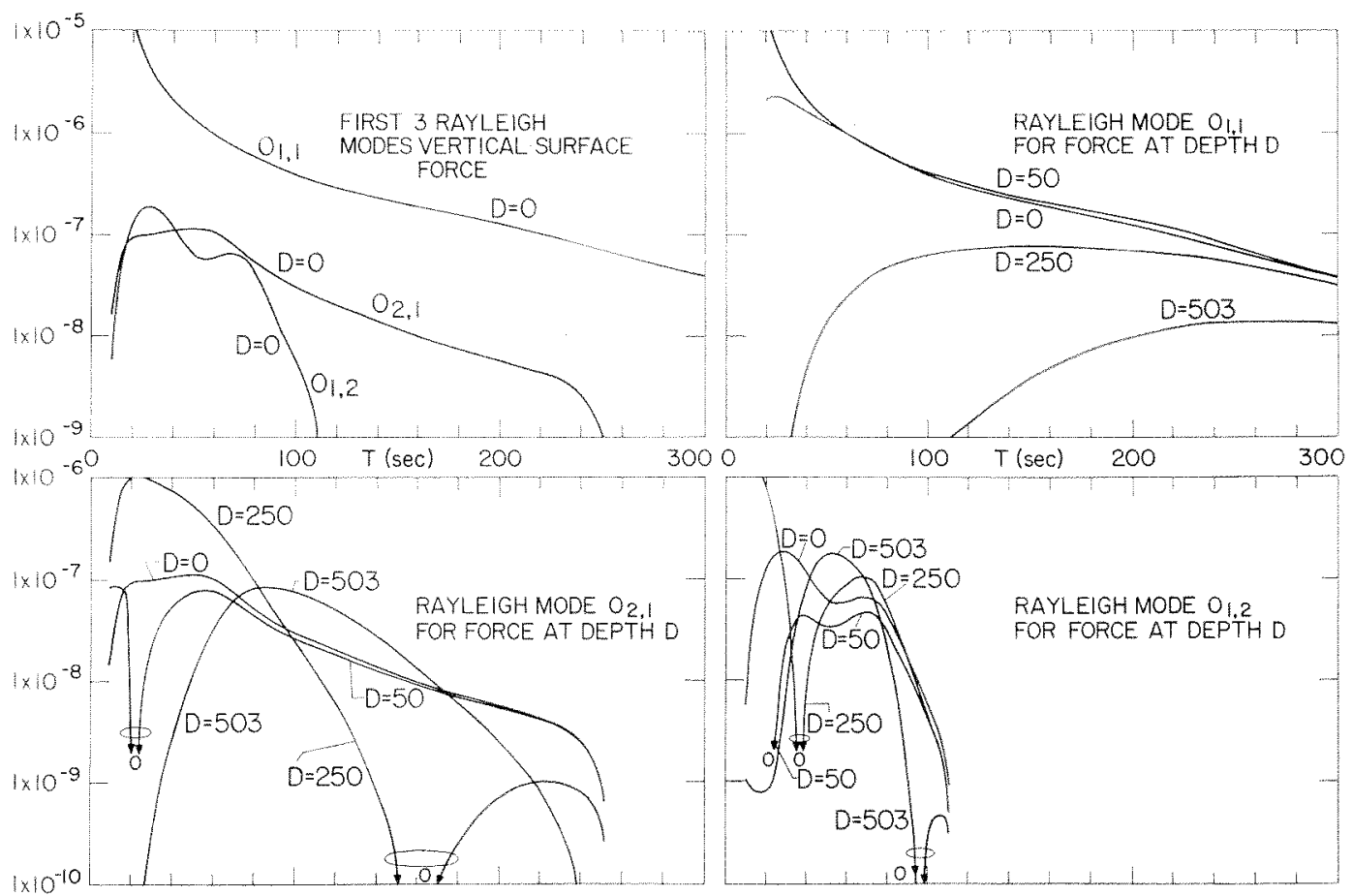

Fig. 7. Rayleigh wave spectrum energy densities per unit oceanic propagation path in units of $10^{\text {ta }} \mathrm{ergs} / \mathrm{km}$ for buried vertical forces of strength $\langle L\rangle=10^{\text {ts }}$ dynes see. 


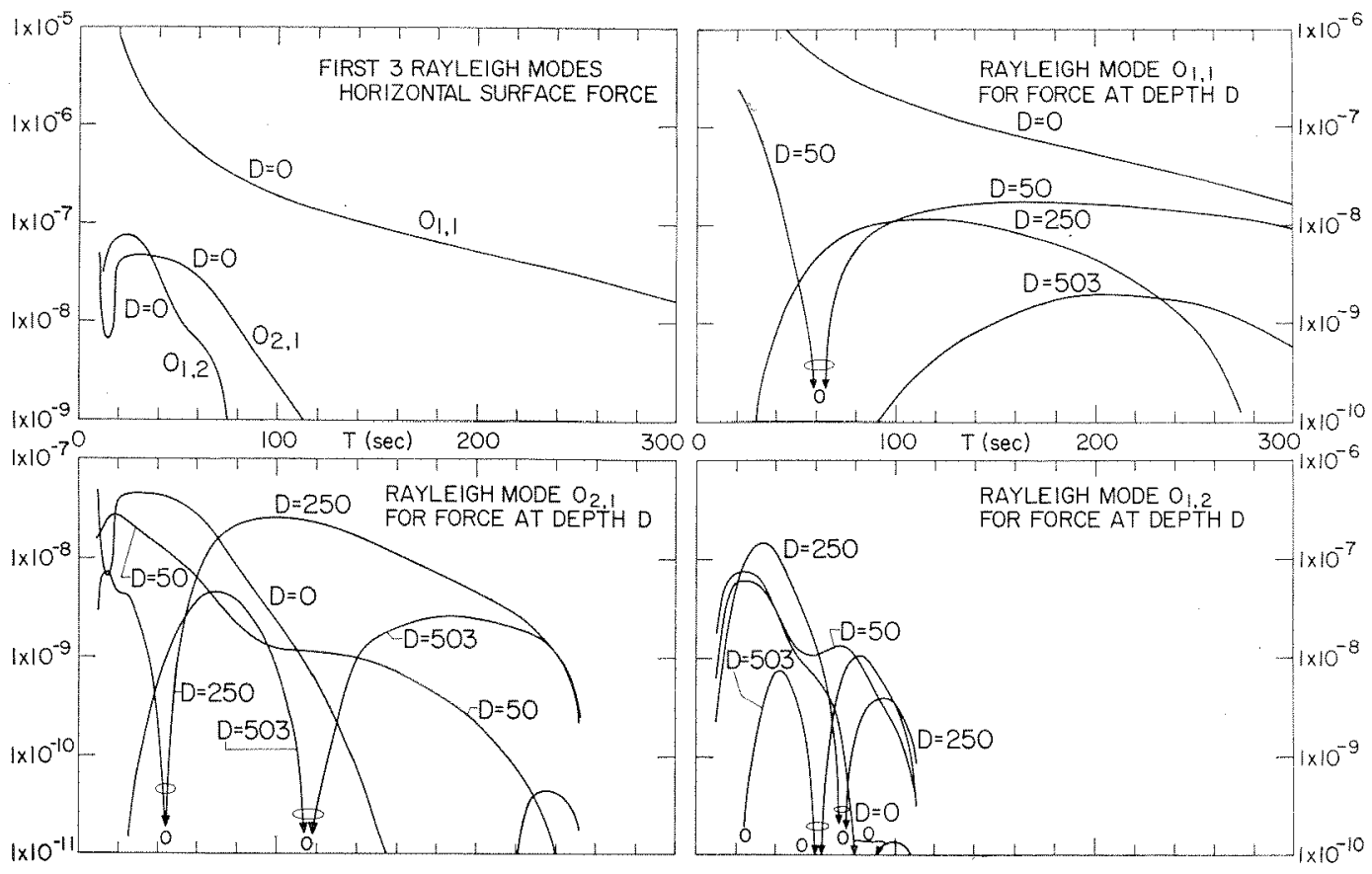

Fig. 8. Rayleigh wave spectrum energy densities per unit oceanic propagation path in units of $10^{15} \mathrm{ergs} / \mathrm{km}$ for buried horizontal forces of strength $\langle L\rangle=2 \times 10^{15}$ dynes sec.
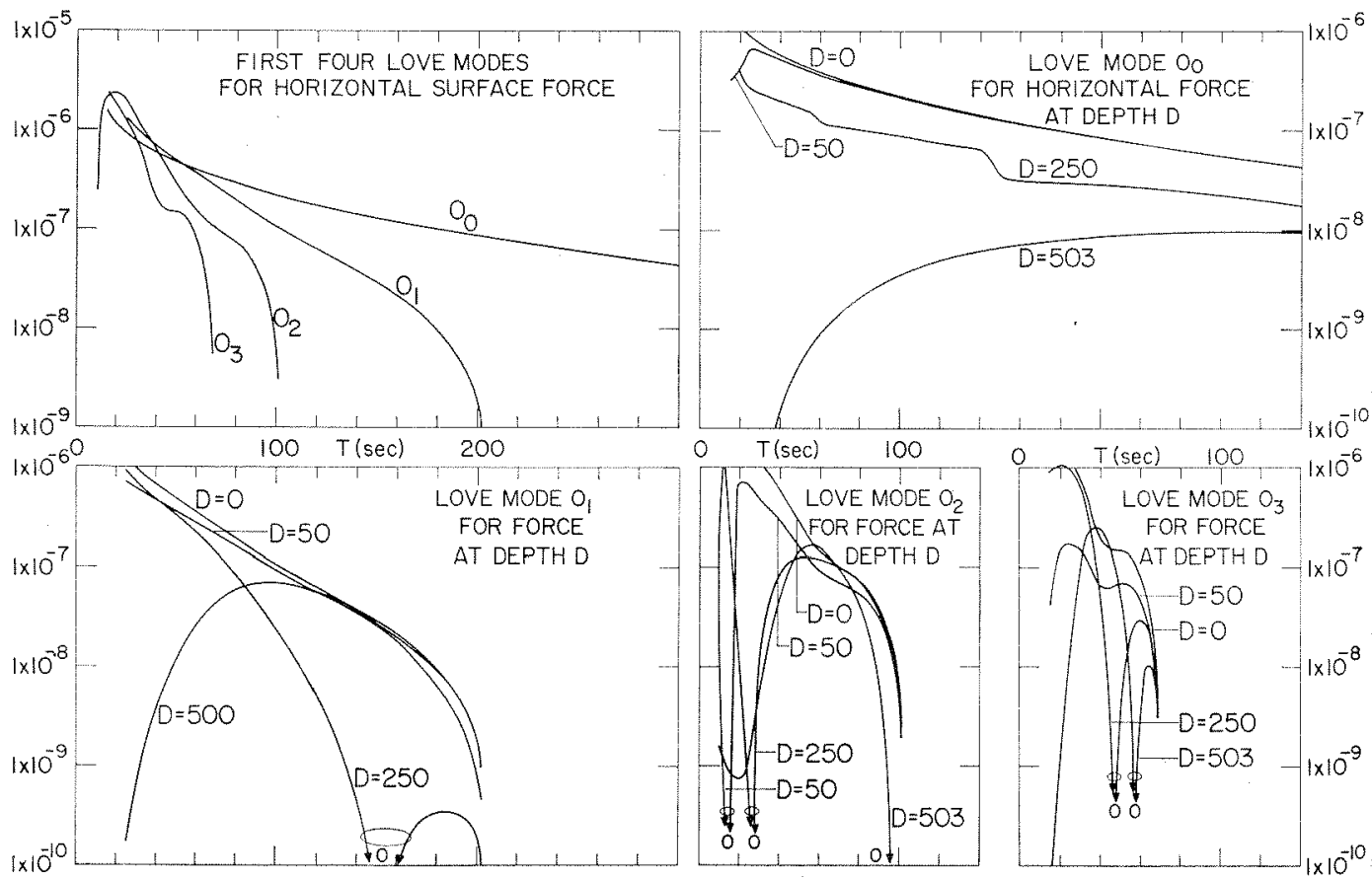

Fig. 9. Love wave spectrum energy densities per unit oceanic propagation path in units of $10^{15} \mathrm{ergs} / \mathrm{km}$ for buried horizontal forces of strength $\langle L\rangle=2 \times 10^{15} \mathrm{dynes}$ see. 
source orientation these zeros are diagnostic of source depth. Note the shift of the zeros with frequency for increasing source depth. The successively deeper sources have zeros at successively longer periods. A source at $250 \mathrm{~km}$, which is in the low-velocity channel, is a very efficient generator of higher-mode waves.

Figure 8 shows the Rayleigh wave energy densities for horizontal forces at various depths. The important difference, for vertical and horizontal forces, is the shift in the location of the energy zeros. The zero locations for the two forces are governed by the nodes of horizontal displacements for horizontal forces and the nodes of the vertical displacements for vertical forces. The most efficient level for the generation of the fundamental mode for periods greater than 20 seconds is at the surface. A source in the channel generates less higher-mode energy than sources at other depths only for periods which involve a vertical displacement node in the channel. This again illustrates the channel wave characteristic of higher modes.

Even though a source in the channel is especially efficient in the generation of higher modes, it should be emphasized that this does not necessarily mean high surface amplitudes. Most of the energy is in the channel. On the other hand, small surface amplitudes of the higher modes do not mean low total energies. One cannot neglect the energy content of higher modes simply because they have low surface amplitudes.

The corresponding Love wave energy densities are displayed in Figure 9. As before, the holes in the energy spectrum of the higher modes are diagnostic of source depth.

\section{CONCLUSIONS}

The tables given in this paper make it possible to convert an observed surface wave ampliwude at a given period to the total energy in the wave at that period, or to convert an observed amplitude spectrum over a certain frequency band to the total energy contained in that band. The figures given here and the tables in Harkvider [1966] make it possible to estimate the partitioning of energy among the various surface wave modes. The use of spectrums and nodal spectral ratios to recover source depth and orientation is discussed further in Hartrider [1966].

To estimate the energy in a frequency band from a seismic source, the data from several stations are needed to define the radiation pattern and source characteristics, which in turn are required for the surface integration. The experimental frequency band should be as wide as possible and, in particular, should include as much information as possible from the shortperiod arrivals. The curves presented here in conjunction with the asymptotic limits can be used in estimating the energy outside the measured band. The energy content of modes not directly analyzed can also be estimated if enough properties of the source are known. The experimental amplitude spectrums should, of course, be corrected for propagation effects such as spreading and attenuation.

Acknowledgment. This research was supported by the Advanced Research Projects Agency and was monitored by the Air Force Office of Scientific Research under contract AF-49(638)-1337.

\section{REFERENCES}

Anderson, Don L., Universal dispersion tables, 1, Love waves across oceans and continents on a spherical earth, Bull. Seismol. Soc. Am., 5it, 681-726, 1964.

Anderson, Don L., Latest information from seismic observations, in The Earth's Mantle, edited by T. F. Gaskell, Academic Press, London and New York, in press, 1966.

Anderson, Don L., and C. B. Archambeau, The anelasticity of the earth, J. Geophys. Res., 69, 2071-2084, 1964.

Anderson, Don L., A. Ben-Menahem, and C. B. Archambeau, Attenuation of seismic energy in the upper mantle, J. Geophys. Res., 7o, 1441$1448,1965$.

Anderson, Don L., and M. N. Toksöz, Surface wave dispersion and structure of the upper mantle, in preparation, 1966.

Archambeau, C. B., and D. L. Anderson, Inversion of surface wave dispersion data, paper presented at IUGG 13th General Assembly, Berkeley, Calif., 1963.

Ben-Menahem, A., and D. G. Harkrider, Radiation patterns of seismic surface waves from buried dipolar point sources in a flat stratified earth, J. Geophys. Res., 69, 2605-2620, 1964.

Harkrider, D. G., Surface waves in multilayered elastic media, 1, Rayleigh and Love waves from buried sources in multilayered elastic half-space, Bull. Seismol. Soc. Am., 54, 627-679, 1964.

Harkrider, D. G., Surface waves in multilayered elastic media, 2, Higher mode spectra and spectral ratios from point forces in plane layered earth models, in preparation for the Bull. Seismol. Soc. Am., 1966.

Haskell, N. A., The dispersion of surface waves in 
multilayered media, Bull. Seismol. Soc. Am. $43,17-34,1953$.

Kellis-Borok, V. I, and T. B. Yanovkaya, Dependence of the spectrum of surface waves on the depth of the focus within the earth's crust, Bull. Acad. Sci. USSR, Geophys. Ser. English Transl., no. 11, 1532-1539, 1962.

Kovach, R. L., and Don L. Anderson, Eigher mode surface waves and their bearing on the structure of the earth's mantle, Bull. Seismol. Soc. Am. 5f, 161-182, 1964.

Jeffreys, $\boldsymbol{H}$., The surface waves of earthquakes, Monthly Notices Roy. Astron. Soc., Geophys. Suppl., 3, 253-261, 1934.

Jeffreys, $H$, Small corrections in the theory of surface waves, Geophys. J., 6, 115-117, 1961.

Meissner, E., Elastiche oberfiächen Querwellen, Verhandl. Intern. Kongr. Tech. Mech., 2nd, Zurich, 3-11, 1926.
Takeuchi, H., M. Saito, and N. Kobayashi, Study of shear velocity distribution in the upper mantle by mantle Rayleigh and Love waves, J. Geophys. Res., 67, 2831-2839, 1962.

Takeuchi, H., J. Dorman, and M. Saito, Partial derivatives of surface wave phase velocity with respect to physical parameter changes within the earth, J. Geophys. Res., 69, 3429-3441, 1964.

Toksöz, M. N., and Don L. Anderson, Phase velocities of long-period surface waves and structure of the upper mantle, 1, J. Geophys. Res., $\% 1,1649-1658,1966$.

Wu, Francis T., Energy of earthquakes, Bull. Seismol. Soc. Am., in press, 1966.

(Manuscript received January $17,1966$. ) 